\title{
Erratum \\ Special Section Introduction: Transnational engagement of Mexican migrant organizations in Chicago
}

Latino Studies (2013) 11, 267. doi:10.1057/lst.2013.11

Correction to: Latino Studies (2013)11, 21-27. doi:10.1057/lst.2012.52

In the previously published version of this introduction the names of two of the co-authors are missing.

The three authors of this introduction are: Judith A. Boruchoff, Stephanie Schütze and Xóchitl Bada. 